Journal of Agriculture and Social Research (JASR) Vol. 8, No. 2, 2008

\title{
DEALING WITH A CONTROLLABLE RISK FACTOR LIKE DIET IN THE MANAGEMENT OF CARDIOVASCULAR DISEASE
}

\author{
ESSIEN, A. \\ Department of Animal Science, \\ Cross River University of Technology, Obubra campus, Nigeria \\ E-mail for correspondence:antighabusola@yahoo.com
}

\begin{abstract}
Cardiovascular disease (CVD) is a silent killer in Nigeria and many parts of the world. Certain factors increase the risk of CVD. While there are controllable factors that contribute and predispose to the development of CVD like diet, exercise, tobacco use, high blood pressure and obesity, there are uncontrollable factors like age, sex and heredity, which are out of the control of the individual. Freedom from CVD is an intricate interaction of many factors. Diet is one strong means of staying free of CVD. This paper posits that rather than calling for a complete boycott of red meat and poultry eggs, thereby throwing those involved in this line of business out of work, professionals involved in animal production, can resort to genetic, dietary, non-dietary, and pharmacological means of controlling cholesterol in meat and eggs. Even though CVD is more common among the aged, artherogenesis (the beginning of artery plaque formation) starts in early childhood. Education is a very important factor in our search for freedom from CVD. Medical personnel, human nutritionists, veterinarians and animal scientists should form a strong synergy in the fight against this scourge.
\end{abstract}

Key words: Cardiovasvcular disease, cholesterol, risk factors, diet.

\section{INTRODUCTION}

Cardiovascular disease is the leading cause of death in the United States (Fahey, 2005).One might want to hastily dismiss this as an American problem until one sees the revelation of Onwuka(2007), who, using data on Waste to Hip ratio(WHR) and Body Mass Index(BMI) of 400 Medical Out Patients(MOP) suffering from cardiovascular disease at the University College Hospital,Ibadan, discovered that the elderly men (60-69 years) and women(50-59 years) fell into the High risk group. But evidence abounds in literature testifying to the fact that atherogenesis (the beginning of artery plaque formation) has its origin in childhood (Iconocast, 2006). Most of the patients that come to clinic are illiterates, which is a major cause for the persistence of diseases (Onwuka, 2007). Nigerians are very secretive when it comes to health matters, coupled with the poverty in the land. Hence they often go to hospital when ailments are at very advanced stages. There are many walking the streets who have cardiovascular disease and are completely oblivious of it. This paper therefore wants to look at Cardiovascular disease, its root causes and likely interventions to keep it under check

Cardiovascular disease (CVD) is simply a disease of the heart and blood vessels (Fahey, 2005).It mostly happens as a result of an interference in the coronary blood flow whereby the blood supply of a portion of the heart is suddenly cut of by an occlusion of one of the large branches of the coronary artery-usually the descending branch of the left coronary(Smith,1972).Coronary occlusion may occur as a result of (1)arteriosclerosis, (2) thrombosis of an already arteriosclerised artery,(3)syphilitic involvement of the mouth of the coronaries and (4)blockage of one of the arteries by an embolus(Smith,1972).Arteriosclerosis is a degenerative process that produces a hardening, thickening and inflexibility of the arterial side of circulation. The inner layers of the arteries are made thick and irregular by deposits of fatty substances often called plaques (Fahey, 2005). The arteries loose their elasticity and their ability 


\section{Journal of Agriculture and Social Research (JASR) Vol. 8, No. 2, 2008}

to expand and contract. Once narrowed by a plaque, an artery is vulnerable to blockage by blood clots. It is a change primarily concentrated in the lining (intima) of the vessels that is designated atherioslerosis. Fibrous tissue proliferation associated with a deposition of fatty substances and calcific salts results in the formation of intimal plaques

Risk factors for cardiovascular disease (CVD): The American Heart Association (AHA) grouped the risk factors into two major categories:(a) the major risk factors and (b) the contributing risk factors. Major risk factors include diet (resulting in unhealthy blood cholesterol), exercise habits (many have sedentary lifestyles), use of tobacco, high blood pressure and obesity. These five risk factors as outlined by the AHA are controllable risk factors. Other risk factors like age, sex and heredity are beyond the control of the individual (AHA, 1998). Predisposition to Chronic heart disease is strongly linked to age, diet, blood pressure, hormones, cholesterol metabolism and several other factors. While genetic counseling of Couples is important as a way out of this problem (arteriosclerosis has been shown to run in families), a closer look at diets that have the unique ability to lower serum cholesterol and other factors that predispose to chronic heart disease is necessary. Some of these factors are discussed below.

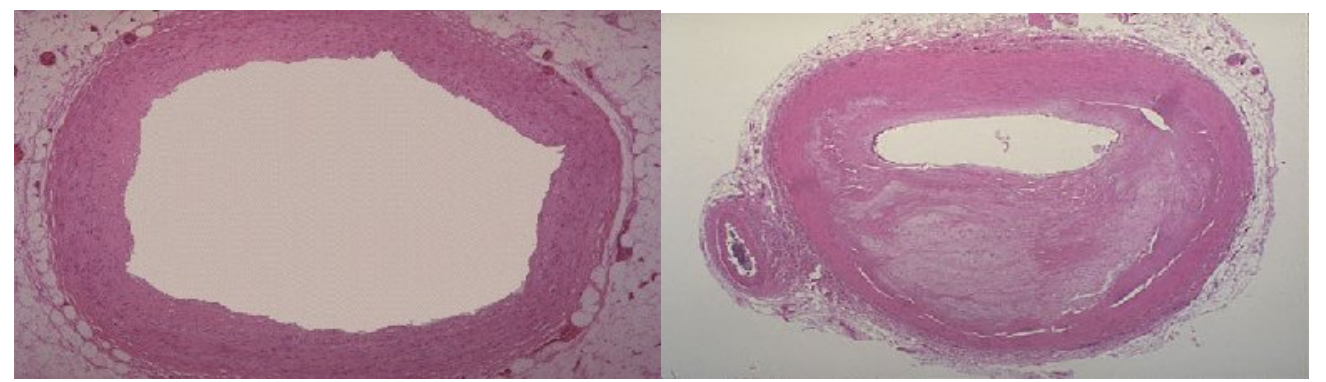

Plate 1:A normal coronary artery with

Plate 2::A coronary artery showing 75\% a big unobstructed lumen.

Occlusion

\section{An elevated total serum homocysteine level:}

An elevated total serum homocysteine (Hcy) level is a well established risk factor for cardiovascular disease(CVD)Elevated Hcy arterial endothelial function, increases oxidative change and promotes inflamation and thrombosis. Strong epidemiological and a number of clinical evidences, have led many to believe that treating elevated Hcy would be important in the prevention of CVD. (Clinical Nutrition updates, 2006; Alfthan ,1997)

A low vitamin status: A low vitamin status is a strong predisposing factor to cardiovascular disease (CVD). It has as a matter of fact, been established by experts that two-thirds of the cases of high Hcy are due to low vitamin status.Hcy status is directly related to the status of the nutrients involved in its metabolism like folate, Vitamins $\mathrm{B}_{12}, \mathrm{~B}_{9}$, and to a less extent, riboflavin(Clinical nutrition update,2006; Hennekens, 1997; Davighis, 1997)).A test was conducted in Germany to determine whether reducing Hcy levels affected coronary artery intima-media thickness. Fifty (50) German patients with intimal-medial thickness of the carotid

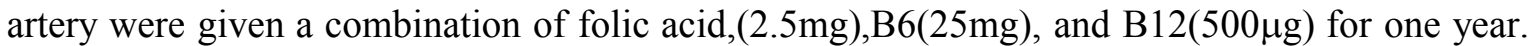
It was found that Hcy reduced from 10.5 to $6.56 \mu \mathrm{mol} / \mathrm{L}$ and carotid intima-medial thickness was reduced by $5.3 \%$. How much of which vitamin is ideal to reduce the Hcy level remains a big question on the lips of researchers. 


\section{Journal of Agriculture and Social Research (JASR) Vol. 8, No. 2, 2008}

An elevated serum cholesterol level: An elevated serum cholesterol level is another strong predisposing factor to heart diseases. There is however, the need to underscore the importance of cholesterol as an important sterol as many writers and researchers have unjustly clamped down on it. Cholesterol is the most important sterol of animal origin, occurring in cellular membranes and body fluids (Ologhobo and Adejumo, 1998)It is an important precursor of steroid hormones, hence playing a pivotal role in sexual reproduction, ensuring the continuation of species and general homeostasis (Hafez,1987). It is associated with the structural components of nerve tissues and there is evidence that dietary cholesterol is deposited in the developing brain (Davidson and Wajda, 1959). Cholesterol is important because it is a constituent of the cell membrane. It is the fluid that coats the lungs and the protective sheaths around the nerves (Roberts, 2000). Its inclusion in semen extenders has been known to increase sperm motility and viability. It is the most important sterol of animal origin occurring in cellular membranes and body fluids (Ologhobo and Adejumo, 1998) Cholesterol is almost inevitable in our diets, besides, the body naturally synthesizes it.

Excess cholesterol is excreted in the bile duct or gall-as gallstones. This sometime blocks the bile duct, leading to obstructive jaundice (where the skin has a yellowish appearance due to the retention of biluribin). Excess cholesterol in the circulating blood may deposit on the walls of the arteries, causing intravasular clot. If this occurs in one of the coronary arteies, it can predispose humans to arteriosclerosis (hypercholesterolemia). Cardiovascular disease which is highly correlated with the amount of cholesterol in the circulating blood is affected by diet (a high dietary cholesterol is a pointer to CVD), liver activities, a high waist to hip ratio, genetic make up of the individual (CVD tends to run more in some families than others), ethnicity, age and gender among other factors(Fang et al,1996; Thun,1997;.McCarron,1997).African Americans have much higher rates of hypertension, heart disease and stroke than other ethnic groups in the United States. This is a risk that blacks all over the world, particularly those in Africa, should take seriously

Cholesterol occurs in the body as lipoproteins (lipoproteins are lipids bounded and carried by protein bodies) and it is manufactured in the liver. They exist as Low Density Lipoproteins (LDL), Very Low Density Lipoprotein (VLDL), Intermediate Low Density Lipoproteins (ILDP) and High Density Lipoproteins (HDL). All these forms of cholesterol have their respective functionalities but with respect to arteriosclerosis, the VLDLs and the LDLs are the major culprits while HDL is good cholesterol, as it helps seek out excess cholesterol, reducing the amount available for building up in the arterial walls (Seidell and Bouchard, 1997;Pond et al, 1995).

Possible control of cholesterol level through diet: Diet is one of the risk factors to CVD. While the exercise physiologist would want to intervene through aerobics and the Public Health experts would be crying out against smoking, animal production experts want to focus on the production of cheap, safe and high quality animal products with good profits in mind. The consumption of red meat has been fingered as a major culprit in the onset and persistence of coronary heart disease (CRD). There have been frantic calls by experts to the citizenry (especially blacks who are at higher risk) to cut down their consumption of red meat and eggs. It is not uncommon to see enlightened men and women that just turned forty, completely change to fish or whole vegetable diets because of the fear of coronary heart disease. This has been a serious threat to the egg industry. Oguntona (1997) captured this in a meeting of stakeholders in the egg industry in Abeokuta, Ogun State, in this statement:

'..the issue of cholesterol has been with the industry for sometime now. As populations become more affluent, educated and more sophisticated, the influence of egg consumption on the health of the individual will continue to be raised...breeding work is going on at an impressive rate in 


\section{Journal of Agriculture and Social Research (JASR) Vol. 8, No. 2, 2008}

Japan and Israel to breed hens that lay very low cholesterol eggs.' The egg still remains the protein source with the highest biological value

Non dietary factors and select pharmacological compounds as egg-cholesterol lowering agents are today being employed in an attempt to lower egg cholesterol. Statins, garlic paste and pharmacological amounts of copper have been orally administered to chickens to achieve egg yolk cholesterol reduction of $46 \%, 32 \%$ and 34\% respectively (Elkin,2007).Phytosterols(plant derived sterols) which are structurally similar and functionally analogous to cholesterol in vertebrates(Ostlund,2002) have been orally administered to treat hypercholesterolemia in chickens over fifty years ago(Peterson,1951).With the recent advances in avian genomics and trangenesis,it is anticipated that the greatest advances in the area of yolk cholesterol reduction will be attained through the manipulation of key genes whose protein products mediate intestinal sterol absorption, hepatic cholesterol and lipoprotein synthesis, and/or lipoprotein uptake by growing oocytes(Eklin,2007).

Udoh(1998) in an experiment to investigate the effect of macerated bitter leaf(Vernonia amygdalina) on the liver and serum lipid profile of fryer rabbits, discovered a general progressive reduction in the serum and liver lipid profile of fryer rabbits as the quantity of macerated bitter leaf meal increased in the diet of the rabbits. Many researchers have also achieved a similar effect with cola. Wether the consumption of meat from rabbits so treated can reduce the risk of CVD is still a green area waiting to be researched.

The effect of Castration on cholesterol level: the physical removal of the testes or the reduction of its functions through non surgical means, thereby terminating the ability of the testes to perform its primary role of producing testosterone, resulting in a general decline in secondary sex characteristics and libido is an age long practice which has been utilized to achieve higher growth rates in farm animals. But a cessation in the production of testosterone translates to an increase in the production of meat with higher levels of total cholesterol (Ladokun 2006). But the consumption of meat from castrated animals being a predisposing factor to CVD is yet to be established empirically.

The effect of testosterone administration on serum cholesterol level: Several researchers have shown that parenteral administration of testosterone can reduce serum cholesterol. Bruck et al, (1997) working with New Zealand White Rabbits and Ladokun,(2006) working with pigs, have shown that testosterone administration can reduce serum cholesterol. This appears to be a great boost to the campaign for the consumption of low cholesterol animal products There is however the need to specify which fraction of serum cholesterol is actually reduced

\section{CONCLUSION}

Cardiovascular disease is a silent killer that has assumed terrible proportions. Controllable and uncontrollable risk factors of CVD have been established. Very little can be done about uncontrollable factors like genetic factors, but controllable factors like taking a healthy, low cholesterol diet is a major assurance against CVD.A call to the citizenry to stop eating red meat and eggs is a serious threat to this sector as many earn their daily income therefrom. Animal production experts should begin to target methods of production that can ensure reduced meat and egg cholesterol. From infancy, a strong consciousness of CVD should be implanted in children, as atherogenesis (the beginning of artery plaque formation) has its origin in childhood. Education is a very important factor in our search for freedom from CRD. Medical personnel, human nutritionists, veterinarians and animal scientists should form a strong synergy in the fight against this scourge 


\section{Journal of Agriculture and Social Research (JASR) Vol. 8, No. 2, 2008}

\section{REFERENCES}

Alfthan, G. 1997. Plasma homocysteine and cardiovascular disease mortality Lancet 349:397.

American Heart Foundation(1998).Heart and stroke statistical update.(retrieved January $25^{\text {th }} 1998$; http://www.americanheart.org/scientific/HSstats98/index.html)

Bruck, B., Brehme, U., Gugel, N., Hanke S., Finking, G., Lutz,C.,Benda,N.,Schmahl,F.W.,Haasis,R and Hanke,H.(1997).Gender-specific differences in the effect of testosterone and estrogen on the development of atherrosclerosis in rabbits

Clinical Nutrition update(2006).Homocysteine and heart disease in 2006.Arbor Clinical Updates 2006(July);1-3, Issue 258.

Daviglus, M.L. 1997. Dietary vitamin C, beta-carotene and 30-year risk of stroke: Results from the Western Electric Study. Neuroepidemilogy 16:69-77.

Davidson,A.N and Wajda,M.(1982).Effect of dietary cholesterol on brain development.J.Neurochem.4,355-361

Elkin,R.G.(2007). Reducing shell egg cholesterol content II.Review of approaches utilizing nonnutritive dietary factors or pharmacological agents and an examination of emerging stratergies.World's Poultry Science Journal, Vol. 63, No.1,(march,2007).

Fahey, T.D (2005).Fit and Well: Core concepts and labs in physical fitness and wellness. Mayfield publishing Company, U.S.A.

Fang, J., .S. Madheaven and M.H.Alderman(1996).The association between birthplace and mortality from cardiovascular causes among black and white residents of New York City. New England Journal of medicine 333(21):1545-1440

Hafez, E.S.E. (1987). Reproduction in farm animals. Lea and Fabiger, 600 Washington square, Philadelphia, U.S.A.

Hennekens, C.H. 1997. Antioxidant vitamins and cardiovascular disease: Current perspectives and future directions. European heart Journal 18:177-179.

Iconoclast (2005). Help Your Kids Healthier. http://www.iconoclast.com/News_files/HNewsXX_XX_Q8/News9a.htm

Ladokun, A, O. (2006). Exogenous Hormonal regulation of growth rate, blood chemistry and fertility in pigs. PhD thesis, Department of Animal Science, University of Ibadan pp 277

McCarron,D.A(1997)..Nutritional management of cardiovascular risk factors.A randomised clinical trial. Archives of internal medicine 157:169-177

Oguntona,E.B.(1997).Effective strategies for egg marketing in Nigeria. Proceedings of workshop organized by the Ogun State Chapter of the Nigerian Society For Animal Production (NSAP) on the $23^{\text {rd }}$ of July.1997 at Gateway Hotel,Abeokuta,Nigeria 
Journal of Agriculture and Social Research (JASR) Vol. 8, No. 2, 2008

Ologhobo,A.D and Adejumo,D.O.(1998) Effect of methionine supplementation and the phospholipids and cholesterol content of the rat brain. Nutrition reports international, Vol.38, No.2.pp 275-281

Onwuka,R.A.(2007).Waste Hip ratio as a risk factor of cardiovascular disease among patients in the medical outpatient(MOP), cardiac clinic, University College Hospital(UCH), Oyo State. B.Sc. Project report, Dept. of Human Nutrition, University of Ibadan.

Ostlund, R.E.(2002). Phytosterols in human nutrition. Annual Review of nutrition 22:533-549

Peterson.D.W.(1951). Effect of soybean sterols in the diet on plasma and liver cholesterol in chicks. Proceedings of the Society for Experimental Biology and Medicine.78:143147

Pond,W.G.,Church,D.C. and Pond,K.R.(1995).Basic animal nutrition and feeding.John Wiley and Sons,New York,U.S.A.

Roberts,M.B.V(2004).Biology: a functional approach(4 ${ }^{\text {th }}$ edition).Thomas Nelson and Sons Ltd/Nelson House Mayfield road/Walton-on thames,KT12,5PL,UK.PP 201-209

Smith, A.L(1972). Diseases of the cardiovascular system. C.M.Mosby 1972. pp500-509

Udoh,M.J(1998).Effect of macerated bitter leaf (Vernonia amygdalina) meal amended rations on the liver and serum lipid profile of fryer rabbits.B.Sc project report, Department of Animal science, University of Calabar. 